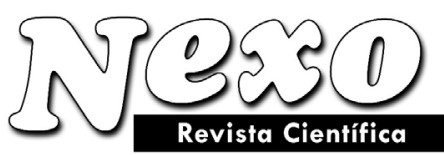

ISSN 1818-6742

Impreso en Nicaragua.

www.nexo.uni.edu.ni

Vol. 23, No. 01, pp.02-08/Mayo 2010

\title{
Variables ambientales y actividades ganaderas determinantes para la Evaluación Ambiental Estratégica de la ganadería bovina en Nicaragua.
}

\author{
J. Gallo \\ Consultor en Gestión Ambiental \\ E-mail:jgallog@gmail.com
}

(Recibido/received: 16-Noviembre-2009; aceptado/accepted: 10-Mayo-2010)

RESUMEN

Como resultado de esta investigación se determinaron las variables ambientales y actividades ganaderas determinantes para la Evaluación Ambiental Estratégica (EAE) de la ganadería bovina en Nicaragua. El estudio permitió definir que las variables ambientales determinantes, por la relación existente entre las actividades ganaderas y antrópicas, están contenidas principalmente en los factores del medio físico construido y medio socioeconómico, siendo estas: el propósito productivo y la integración de la ganadería con los cultivos y pastos. A su vez, las actividades ganaderas se relacionan directamente con las dimensiones técnicas propias del sector ganadero, así como de los aspectos sociales y económicos, resultando la de mayor valor, la alimentación del ganado. Las variables y actividades ganaderas determinantes, permitieron definir el inicio del proceso de evaluación y constituyeron una herramienta para planificar y ejecutar acciones para el desarrollo del sector ganadero en el país, y a su vez, representaron un equilibrio para la relación sostenible entre los factores ambientales y el sector mismo. La formación de paneles de expertos y el empleo de métodos matemáticos fueron necesarios para la selección de las variables y actividades.

Palabras claves: variables ambientales, evaluación ambiental estratégica, ganadería bovina.

\begin{abstract}
As a result of this research, environmental variables and cattle raising were determined by the strategic environmental evaluation of cattle raising in Nicaragua. The study allowed for the definition and determination of environmental variables and the current relationship between cattle raising and anthropic. The principal components of the factors in the physical and socio-economic environments, these being: The production and integration of cattle raising with crop production and grazing land (pasture). At the same time, cattle raising is directly related to the techniques used in the cattle industry as well as social and economic aspects resulting in improved cattle feed. The variables and cattle raising determined allow for definition at the beginning of the evaluation process and constitute a tool for planning and action for the development of the country's cattle industry. At the same time it represents a balance of the sustainable relationship between environmental factors and the industry itself. The creation of panels of experts and the use of mathematical methods will be necessary for the selection of variables and activities.
\end{abstract}

Keywords: enviromental variables, strategic environmental assessment, cattle raising. 


\section{INTRODUCCIÓN}

Nicaragua es un país que fundamenta su economía en la actividad agrícola y agropecuaria. Dentro de este escenario, el sector ganadero aporta a la nación un importante componente económico, social y ambiental (Nicaragua en Cifras, 2010). A pesar de esto, el rubro ganadero en la actualidad posee sistemas tecnológicos poco desarrollados y de reducida productividad.

Al sector agropecuario y concretamente a la ganadería bovina se le relaciona constantemente con la generación de desequilibrios ambientales en las zonas geográficas en donde se desarrolla. Tal como lo cita el Informe estado del ambiente en Nicaragua (2001), uno de los principales problemas ambientales del país es la expansión territorial de la ganadería y agricultura (continuo avance de la frontera agrícola), la cual está causando de forma directa la desaparición de los bosques, principalmente en la región Atlántica ubicada al este del país. En Nicaragua la frontera agrícola avanza en una sola dirección: de oeste a este, ya que dicha región es la única que aún conserva bosques vírgenes de grandes extensiones propiedad de la nación.

La mayoría de los problemas ambientales generados por las actividades agropecuarias, pueden ser identificados tempranamente. En el caso de los programas y proyectos del sector agropecuario, los problemas son originados desde su formulación -de los planes y programas- ya que estos frecuentemente no incorporan las consideraciones ambientales concernientes a la actividad.

En este estudio se introdujo una herramienta, que permitió incorporar la dimensión ambiental a un sistema de planificación, conocida como la Evaluación Ambiental Estratégica (EAE). Esta herramienta consistió en un proceso de carácter preventivo, sistemático, integrador y global, que evalúa las posibles repercusiones ambientales de las propuestas de políticas, planes y programas durante su proceso de elaboración, integrando las consideraciones ambientales en los procesos de toma de decisiones estratégicas, por medio de la incorporación de los objetivos ambientales además de los económicos y sociales. Este proceso requiere de la participación de los actores del tema a evaluar (Gallo, 2009).

La metodología de la EAE debe ser diseñada en cada país debido a que cada uno de ellos posee sus propias condiciones económicas, sociales, políticas, culturales y ambientales (Oñate, 2002). Es decir, cada país debe desarrollar su propia metodología para identificar y definir las variables para la elaboración de su particular EAE.

Esta investigación propone la determinación de tales variables para la Evaluación Ambiental Estratégica, en una actividad económica de gran importancia para el país, como lo es el sector ganadero.

\section{MATERIALES Y MÉTODOS}

Los materiales empleados en esta investigación fueron:

- Documentación electrónica: extraída de bases de datos calificados.

- Documentación impresa: libros, revistas.

- Entrevistas personales.

Los métodos utilizados en esta investigación fueron:

1. Análisis bibliográfico de la información recopilada.

2. Análisis matricial de interacción elaborada y aplicada por el comité de expertos mediante el uso de las siguientes herramientas:

- Grupo focal: El desarrollo de esta herramienta metodológica, permitió la identificación de las variables y actividades más significativas para su posterior análisis, la determinación final de las variables y de las actividades que forman parte de la Metodología de Evaluación Ambiental Estratégica.

- Entrevista personal: A través de éstas, se logró establecer un determinado número de variables y actividades propias del sector ganadero.

1. Análisis Bibliográfico.

El análisis bibliográfico consistió en la clasificación y sistematización de la información relacionada con la evaluación ambiental estratégica de la ganadería bovina. 


\section{Análisis matricial por el comité de expertos}

La determinación de las variables y actividades ganaderas más determinantes para la EAE fue realizada por un grupo de expertos empleando un análisis de matriz de interacción.

\section{Comité de expertos:}

Durante la realización de esta investigación fue indispensable crear un comité de expertos, cuyos objetivos fueron acompañar y validar la investigación. La retroalimentación con el comité de expertos fue desarrollada a través de los grupos focales.

Korman (1992), define a los grupos focales como "la reunión de un grupo de individuos seleccionados por los investigadores para discutir y elaborar, desde la experiencia personal, una temática o hecho social que es objeto de investigación".

De acuerdo con esta definición, desde el inicio de la investigación se seleccionó al grupo de expertos, el cual está compuesto por personas con vastos conocimientos en el tema de la ganadería y la evaluación ambiental, y a su vez representan a instituciones públicas y privadas relacionadas con el tema investigado.

Este comité de expertos fue integrado por miembros del Sector Público Agropecuario (SPAR), compuesto por: el Instituto de Desarrollo Rural (IDR), Ministerio del Ambiente y los Recursos Naturales (MARENA), Ministerio Agropecuario y Forestal (MAGFOR), Instituto Nicaragüense de Tecnología Agropecuaria (INTA), productores ganaderos representantes de gremios organizacionales afiliados a la Cooperativa de Servicios Múltiples (COOPROMUSUN) y Cooperativa de Servicios Múltiples de Paiwas (COOSEMUPA), miembros de ONG, docentes investigadores de la Facultad de Ciencia Animal de la Universidad Nacional Agraria (UNA), y el Instituto Interamericano de Investigación Agrícola (IICA).

Con el grupo de expertos se realizaron talleres sobre la dinámica de trabajo, la importancia y objetivos a alcanzar con las sesiones de trabajo del Grupo Focal. Estas sesiones permitieron que los participantes expresaran sus criterios y puntos de vista particulares, y que a posteriori condujeran a que se alcanzaran consensos comunes. Posteriormente se desarrollaron sesiones de entrevistas personales con los participantes, con las que se lograron obtener más elementos de consulta para la identificación de las variables y actividades determinantes para la elaboración de la Evaluación Ambiental Estratégica en la ganadería bovina.

Tomando como referencia los resultados obtenidos del proceso de consulta para la identificación de variables y actividades claves del sector ganadero, realizado con expertos, se propuso:

a) Las variables relacionadas con el entorno ambiental, agrupadas en el medio físico construido, medio físico natural y el medio socioeconómico.

b) Las actividades que constituyen la ganadería tradicional del país.

Posterior a estos procesos, las variables y actividades ganaderas se sometieron a un análisis matricial.

\section{Análisis matriz de interacción:}

Una vez listadas las variables y actividades, se procedió a realizar el análisis para la identificación y la selección de las mismas, en razón de cuáles eran más determinantes para el sector. Para este caso se utilizó el método matricial de interacción entre variables y actividades (éste método fue propuesto y valorado por el comité de expertos). Empleando para esto el programa Microsoft Excel (ver Tabla 1). Se ubicaron las variables en las filas y las actividades en las columnas.

En dicho proceso de análisis, se tomaron como escala los rangos de valoración de cero a tres (0-3), que se constituyen como parámetros a utilizar en la tabla de interacción -variables vs actividades-, de la siguiente forma:

Alta relación entre variable $1 \mathrm{y}$ actividad A: 3 Media relación entre variable 1 y actividad A: 2 Baja relación entre variable 1 y actividad A: 1 No hay relación entre variable 1 y actividad A: 0

El método matricial es ilustrado en la Tabla No. 1, que se muestra a continuación: 
Tabla 1 Matriz de interacción entre actividades ganaderas y variables ambientales.

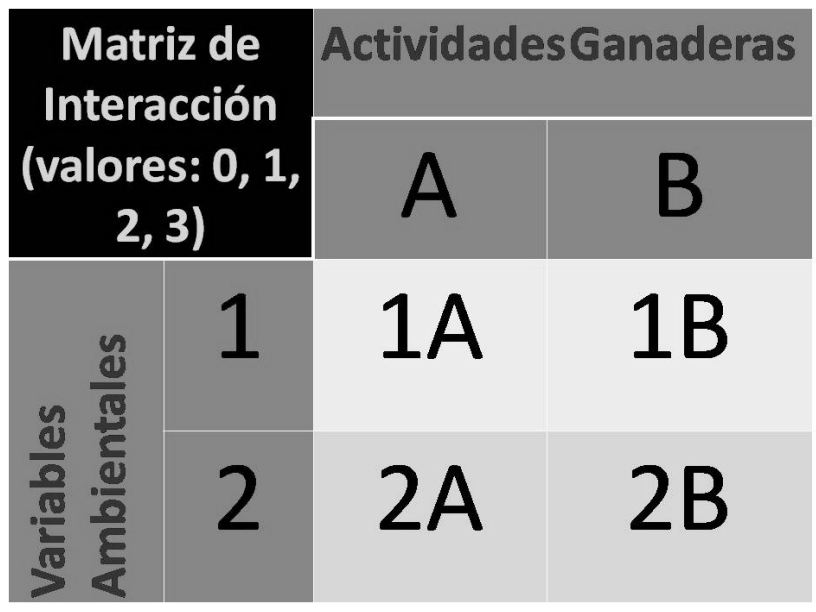

Al completar la matriz con los valores, se obtuvieron los siguientes resultados.

\section{RESULTADOS Y DISCUSIÓN}

En una etapa posterior a la determinación del valor de cada relación, se calculó el promedio de todos ellos, para este estudio fue de 1.62. Este valor promedio, por tanto pasó a constituirse en un valor de discriminación.

De igual forma se obtuvieron los valores promedios de la relaciones de cada variable con las actividades (promedio de los valores de cada fila), y el valor promedio de cada actividad con las variables (promedio de los valores de cada columna). Los valores promedios de las variables $\mathrm{y}$ de las actividades que superaron el valor del discriminante (1.62), fueron categorizadas como variables $\mathrm{y}$ actividades más determinantes.

Tabla 2 Variables claves para la EAE.

\begin{tabular}{|c|c|c|c|c|c|c|}
\hline \multicolumn{3}{|c|}{ Variables } & $\begin{array}{c}\text { Valor de } \\
\text { Import ancia }\end{array}$ & \multicolumn{3}{|c|}{ Indicador } \\
\hline \multirow{8}{*}{$\begin{array}{l}\text { Fisico } \\
\text { Construido }\end{array}$} & \multirow{2}{*}{$\begin{array}{l}\text { Pastos y } \\
\text { forraie }\end{array}$} & Calidad de pasto $y$ forraje & 2.28 & \multirow{2}{*}{$\begin{array}{l}\text { Disponibilidad de alimento (pa sto y forraje) } \\
\text { de buena calidad durante todo el año }\end{array}$} & \multirow{13}{*}{\multicolumn{2}{|c|}{ Capacidad de uso de suelo }} \\
\hline & & Disponibilidad de pasto $\mathrm{r}$ forraje & 2.24 & & & \\
\hline & \multirow{4}{*}{$\begin{array}{l}\text { Ganado } \\
\text { Bovino }\end{array}$} & Raza del ganado & 2.24 & \multirow{4}{*}{$\begin{array}{l}\text { Concordancia entre la raza de l } \\
\text { ganado, salud, proposito productivo y la } \\
\text { ubicación geografica }\end{array}$} & & \\
\hline & & Salud del ganado & 1.79 & & & \\
\hline & & \begin{tabular}{|l|} 
Proposito productiva \\
\end{tabular} & 2.52 & & & \\
\hline & & Ubicación ge ografica & 1.83 & & & \\
\hline & Cultivo & Integración y diversificación productiva & 2.34 & Integración $\gamma$ diversificación productiva & & \\
\hline & \multicolumn{2}{|l|}{ Infraestructura } & 2.31 & Disponibilidad de Infrae structura & & \\
\hline \multirow{5}{*}{ Físico Natural } & \multirow{2}{*}{ Suelo } & Calidad & 1.62 & Calidad del suelo & & \\
\hline & & Uso de la tierra & 1.62 & Uso de la tierra & & \\
\hline & \multirow{3}{*}{\begin{tabular}{|l|} 
Hidrología e \\
Hidrogeología \\
Clima
\end{tabular}} & Calidad & 1.93 & \multirow{2}{*}{$\begin{array}{l}\text { Disponibilidad de agua de buena calidad } \\
\text { durante todo el año }\end{array}$} & & \\
\hline & & Disponibilidad & 2.28 & & & \\
\hline & & Hume dad Relativa & 1.62 & Humedad Relativa & & \\
\hline \multirow{11}{*}{ Socioeconomico } & \multirow{3}{*}{ Social } & Tecnología & 2.10 & Nivel tecnologico & $\begin{array}{r}\text { Apropiación de la } \\
\text { transferencia de tecnología }\end{array}$ & Gobernanza \\
\hline & & Calentamiento global & 1.76 & Emisión de gases de efecto invernadero & \multicolumn{2}{|c|}{ Emisión de gases de efecto inv erna dero } \\
\hline & & Frontera Agrícala & 1.86 & $\begin{array}{l}\text { Cambio en cobertura fore stal en sistemas } \\
\text { ganaderos }\end{array}$ & \multicolumn{2}{|c|}{$\begin{array}{r}\text { Cambia en cobertura forestal en sistema s } \\
\text { ganaderos }\end{array}$} \\
\hline & \multirow{2}{*}{$\begin{array}{l}\text { Recursos } \\
\text { Human 0s }\end{array}$} & Técnico & 1.93 & Asistencia Técnica & \multicolumn{2}{|c|}{ Asistencia Técnica } \\
\hline & & Fuerza de trabajo & 1.86 & Disponibilidad de ma no de obra & \multirow{2}{*}{\multicolumn{2}{|c|}{ Gobernanza }} \\
\hline & \multirow{2}{*}{ Economia } & Tenencia de la tierra & 1.72 & Sequridad de la tierra & & \\
\hline & & Ingresos & 1.86 & Nivel de ingresos de los ganaderos & \multicolumn{2}{|c|}{ Acceso a lnversiones } \\
\hline & \multirow{2}{*}{ Cultural } & Capacidad Técnica del productor & 2.03 & Nivel técnico del productor & Apropiación de la & \multirow{3}{*}{ Gobernanza } \\
\hline & & Cultura del productor & 2.17 & Nivel cultural del productar & transferencia de tecnología & \\
\hline & \multirow{2}{*}{ Institucional } & $\begin{array}{l}\text { Organizaciones y Delega ciones } \\
\text { Institucionales en los territorios }\end{array}$ & 1.72 & \multicolumn{2}{|l|}{ Nivel Organizacional e Institucional } & \\
\hline & & Planes, Programas y Proyectos & 1.79 & $\begin{array}{l}\text { Ejecución de planes, programas y } \\
\text { provectos }\end{array}$ & Acceso & inversiones \\
\hline
\end{tabular}


Como resultado de este proceso se obtuvo que las principales variables del sector ganadero están distribuidas en los factores del medio físico construido, físico natural y socioeconómico, siendo la variable de mayor valor, el propósito productivo de la ganadería y la de menor la humedad relativa.

Las variables fueron agrupadas y caracterizadas de acuerdo con aspectos comunes:

La capacidad de uso del suelo agrupó las variables: suelo, agua, clima, infraestructura, ganadería y su integración con los cultivos o diversificación. Esta nueva unidad de análisis permite determinar qué suelo y bajo qué circunstancias son aptas para la ganadería.

El cambio en cobertura forestal permitió determinar el avance de la frontera agrícola en una región determinada. Se ha comprobado que la ganadería está íntimamente relacionada al avance de la frontera agrícola, por lo tanto se constituye en un indicador de gran relevancia en lo referente a la medición de los impactos negativos de la actividad ganadera.

La asistencia técnica se refiere a la asistencia que recibe un productor agropecuario por parte de las instituciones públicas, privadas o de su propia organización. Esta asistencia es influenciada muchas veces por la ejecución de planes, programas y proyectos.
La gobernanza, se refiere a la administración de las gestiones efectuadas por los productores. Esta abarca lo referente a la fuerza de trabajo, la seguridad de la tierra, la capacidad técnica y cultural del productor y a su organización.

El cambio climático, es el resultado de la preocupación social que existe referente a este fenómeno, y cómo la actividad ganadera aporta en la generación de los gases del efecto invernadero. Desde el punto de vista de los productores, el acceso a fuentes de financiamiento, es el indicador más determinante, no obstante, conviene destacar que las variables deben analizarse de manera integral. Lo que representa entenderlas como elementos que integran un sistema, es decir, unas son complementarias de las otras y se apoyan entre sí, y se sustentan para generar un equilibrio del sector ganadero.

Determinadas las variables, fue necesario conocer las actividades más determinantes del sector ganadero.

Tabla 3. Actividades ganaderas claves para la EAE.

\begin{tabular}{|c|c|c|c|}
\hline \multicolumn{3}{|r|}{ Actividades } & $\begin{array}{c}\text { Valor de } \\
\text { importancia }\end{array}$ \\
\hline \multirow{7}{*}{ Alimentación } & \multirow{5}{*}{$\begin{array}{l}\text { Pastos y } \\
\text { Forraje }\end{array}$} & Preparacion de suelo y siembra de pastos y forraje & 1.83 \\
\hline & & Manejo del pasto y forraje & 1.93 \\
\hline & & Elaboración y almacenamiento de heno o ensilaje & 1.68 \\
\hline & & Establecimiento y manejo de bancos de proteinas & 1.78 \\
\hline & & Establecimiento y manejo de sistemas silvospastoriles & 1.93 \\
\hline & \multirow{2}{*}{ Concentrado } & Preparacion de suelo y siembra del cultivo & 1.90 \\
\hline & & Manejo del cultivo & 1.85 \\
\hline \multirow{3}{*}{\multicolumn{2}{|c|}{ Infraestructura e Instalaciones }} & Construccion y mantenimiento de cercas y corrales & 1.65 \\
\hline & & $\begin{array}{l}\text { Construccion y mantenimiento de galeras y salas de } \\
\text { ordeño }\end{array}$ & 1.68 \\
\hline & & $\begin{array}{l}\text { Construccion, mantenimiento y proteccion de fuentes } \\
\text { de agua }\end{array}$ & 1.65 \\
\hline \multirow{2}{*}{\multicolumn{2}{|c|}{ Ganado Bovino }} & Pastoreo (rotacion de potreros) & 2.18 \\
\hline & & Estabulacion & 2.08 \\
\hline \multirow{2}{*}{\multicolumn{2}{|c|}{ Produccion }} & Leche (ordeño y engorde) & 1.95 \\
\hline & & Procesamiento de leche & 1.70 \\
\hline \multirow{3}{*}{\multicolumn{2}{|c|}{$\begin{array}{l}\text { Comercializacion } \\
\text { Aspectos sociales y } \\
\text { economicas }\end{array}$}} & Comercializacion de leche y carne & 1.68 \\
\hline & & Organización & 1.63 \\
\hline & & Fuentes de financiamiento & 1.63 \\
\hline
\end{tabular}


Como resultado del proceso se obtuvieron las principales actividades del sector, las que están inmersas tanto en las dimensiones técnicas propias de la actividad, como en los aspectos sociales y económicos, siendo la de mayor valor el pastoreo del ganado y la de menor valor la construcción y mantenimiento de las cercas de corrales.

La variable manejo del sistema no se incluyó dentro del análisis matricial, ya que es una actividad que es aplicada de manera transversal a todas las variables y actividades ganaderas, por lo tanto su análisis debe ser intrínseco.

Así mismo, de este análisis se determinó que existe una integración entre la ganadería y los cultivos, (por lo tanto lo mejor es analizar de forma integrada ambos factores).

Estas resultaron ser las principales variables y actividades en el sector de la ganadería bovina en el país, por lo que deberán estar incluidas durante el proceso de elaboración de los planes y programas del sector ganadero.

\section{CONCLUSIONES}

La determinación de las variables y actividades determinantes para la Evaluación Ambiental Estratégica es el punto de partida para la planificación y evaluación paralela de los planes, programas y políticas ganaderas.

Este análisis matricial muestra que las variables y actividades deben ser estudiadas como sistemas de interrelaciones (análisis sistémico), lo que garantiza el equilibrio del mismo.

Los comités de expertos son una herramienta de gran utilidad para la obtención de información y la validación de la misma.

La ganadería, por la generación de gases de efecto invernadero está vinculada directamente con el cambio climático, y representa un reto para los actores locales mejorar el manejo del ganado y contribuir de esta forma a mitigar los efectos que producirá el cambio climático.

\section{REFERENCIAS}

Aguilar, G. (2003). Evaluación de Impacto Ambiental para Centroamérica. Tomo 3 Evaluación Ambiental Estratégica. Ministerio de Asuntos Exteriores de los Países Bajos. San José, Costa Rica

Banco Central de Nicaragua, (2010). Nicaragua en Cifras. Managua, Nicaragua.

Belli, R., et al. (2006). El Desarrollo Ganadero en Nicaragua y su influencia sobre: El Bienestar Socioeconómico de las Familias, la Biodiversidad y los Servicios Ambientales. Universidad Centroamericana. Managua, Nicaragua

Bertilsson, J. (2002). Methane emissions from enteric fermentation-effects of diet composition. En: Petersen, S.O., Olesen, J. (Eds.), Greenhouse Gas Inventories for Agriculture in the Nordic Countries. 81. pp 37-45.

Food and Agriculture Organization (FAO). (2002). Bienvenido a la Caja de Herramientas para Zonas de Ladera Sobre Ganadería y Ambiente. Proyecto LEAD (Livestock, Environment and Development Initiative). Disponible en: www.lead.virtualcentre.org.

Gallo, J. (2009). Instrumento para la Evaluación Ambiental Estratégica de la Ganadería Bovina en Nicaragua. Tesis Doctoral. Managua, Nicaragua.

Jongebreur, A. (2000). Strategic Themes in Agricultural and Bioresource Engineering in the 21st Century. J. agric. Engage Res. 76, pp. 227-236 disponible en: http://www.idealibrary.com

Korman, (1992). Citado por Aigneren, Artículo publicado en CEO, Revista Electrónica no. 7 Medellín, 2000. Disponible en: http://huitoto.udea.edu.co/co. La técnica de recolección de información mediante grupos focales.

Ministerio del Ambiente y de los Recursos Naturales, (2001). Informe Estado del Ambiente en Nicaragua. 2001. Managua, Nicaragua. 
Nicholson, C., et al. (2001). Environmental Impacts of Livestock in the Developing World.

Oñate, J., et. al. (2002). Evaluación Ambiental Estratégica. p-60. Ediciones Mundi-Prensa. Madrid, España

Partidario, M. \& Arts, J. (2005). SEA Follow-up. Exploring the concept of strategic environmental assessment follow-up. Impact Assessment and Project Appraisal, Beech Tree Publishing, 23(3), pp. 246-257.

Rodrigues, G., Campanhola, C. y Choji, P. (2003). An environmental impact assessment system for agricultural R\&D. Environmental Impact Assessment Review 23. pp. 219-244.

Stinchcombe, K. \& Gibson, R. (2001). Strategic Environmental Assessment as a means of pursuing sustainability: ten advantages and ten challenges. Journal of Environmental Assessment Policy and Management (JEAPM). 3(3). pp. 343-372.

Verheem, R. y Jamn, T. (2000). Strategic environmental assessment: one concept multiple forms, Impact Assessment and Project Appraisal, 18 (3).
Verheem, R. (2003). Evaluación Ambiental Estratégica. San José, Costa Rica: Oficina Regional para Mesoamérica.

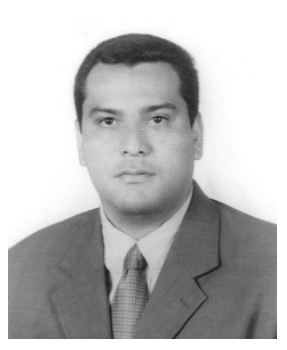

Jorge Gallo, se graduó de Ingeniero Agrónomo, en El Zamorano en 1997. Obtuvo los grados científicos de Máster en Planificación y Administración Ambiental de Proyectos (2006) y Doctor en Ciencias del Ambiente (2009), ambos en el Programa de Estudios Ambientales Urbanos y Territoriales (PEAUT) de la Universidad Nacional de Ingeniería (UNI). Actualmente es Coordinador Técnico del Doctorado Ciencia Tecnología y Desarrollo de la UNI. Su área de investigación es la ciencia, tecnología, planificación, la evaluación y los proyectos y negocios rurales. 